\title{
A Rare Tumor of the Nasal Cavity: Hemangiopericytoma
}

\author{
Kaustubh J. Kahane ${ }^{1}$ Siddhartha Shrivastava ${ }^{1}$ \\ ${ }^{1}$ Department of ENT and Head Neck Surgery, Postgraduate \\ Institute, Yashwantrao Chavan Memorial Hospital, Pimpri, \\ Pune, India
}

Int J Recent Surg Med Sci 2020;6:38-40

\author{
Pune, India
}

\author{
Apurva A. Jarandikar ${ }^{1}$ Aishwarya S. Phatak
}

\begin{abstract}
Address for correspondence Siddhartha Shrivastava, DLO, DNB, ENT, Department of ENT and Head Neck Surgery, Postgraduate Institute, Yashwantrao Chavan Memorial Hospital, Sant Tukaram Nagar, Pimpri, Pune 411018, Maharashtra, India (e-mail: drsshrivastava@gmail.com).
\end{abstract}

\section{Introduction}

Hemangiopericytomas (HPCs) are uncommon vascular tumors originating from the extracapillary cells called pericytes, and they rarely occur in the nose or paranasal sinuses. ${ }^{1}$ The treatment of choice is a wide surgical excision. A high incidence of local recurrence (8-53\%) and metastasis (35-57\%) has been reported., ${ }^{1,2}$ There is no consensus about the efficacy of radiotherapy or adjuvant chemotherapy. ${ }^{2}$ The role of immunotherapy has not been as well known.

Recombinant interleukin-2 (rIL-2: Imunase, Shionogi Pharmaceutical Co., Ltd., Osaka, Japan) is one of the cytokines produced from lymphocytes of the human spleen by genetic engineering. ${ }^{3}$ It has been reported that natural killer (NK) cells amount to 15 to $20 \%$ of all lymphocytes, and decreased NK cell activity was shown in many patients with malignant tumors. ${ }^{3}$ rIL-2 administration demonstrated not only activated NK cells but also antitumor effects in in vitro experiments. ${ }^{4}$ Clinically, the safety and efficacy of rIL-2 have been reported for malignant vascular tumors. ${ }^{5}$ However, the efficacy of rIL-2 against HPC has not been established.

In this report, we describe a rare sinonasal tumor, benign nasal HPCs, which are derived from capillary pericytes and successfully treated with endoscopic surgical excision.

\section{Case Report}

A 57-year-old man with a chief complaint of left nasal obstruction and nasal bleeding was referred to our department. Nasal endoscopy showed a mass between the left nasal septum and the inferior turbinate (-Fig. 1). Contrastenhanced computed tomography (CT; nose and paranasal sinuses) revealed a heterogeneous mass lesion $(5 \times 4 \mathrm{~cm})$ with an enhancement effect that filled the left nasal cavity (-Fig. 2). The nasal septum and the lateral nasal wall were not deformed. Soft tissue density was observed in the bilateral ethmoid sinus as well as bilateral maxillary sinus, but it was thought to be a mucous thickness due to its difference from the tumor's density. The tumor evidenced an equivalent signal in the T1- and T2-weighted images of magnetic resonance imaging (MRI) with a strong enhancement effect.

After all routine examinations as well as complete nose examination, the patient was planned for diagnostic nasal endoscopy, in which we noticed that the mass had its origin from the lateral wall of the left nasal cavity with mucous secretions in both the nasal cavities (-Fig. 3). A biopsy specimen was taken, and the postbiopsy hemorrhage was controlled by endonasal gauze packing. The tumor was proved to exhibit a benign HPC histopathologically.
DOI https://doi.org/

10.1055/s-0040-1713563

ISSN 2455-7420.
License terms

() (1) $\ominus \circledast$ 

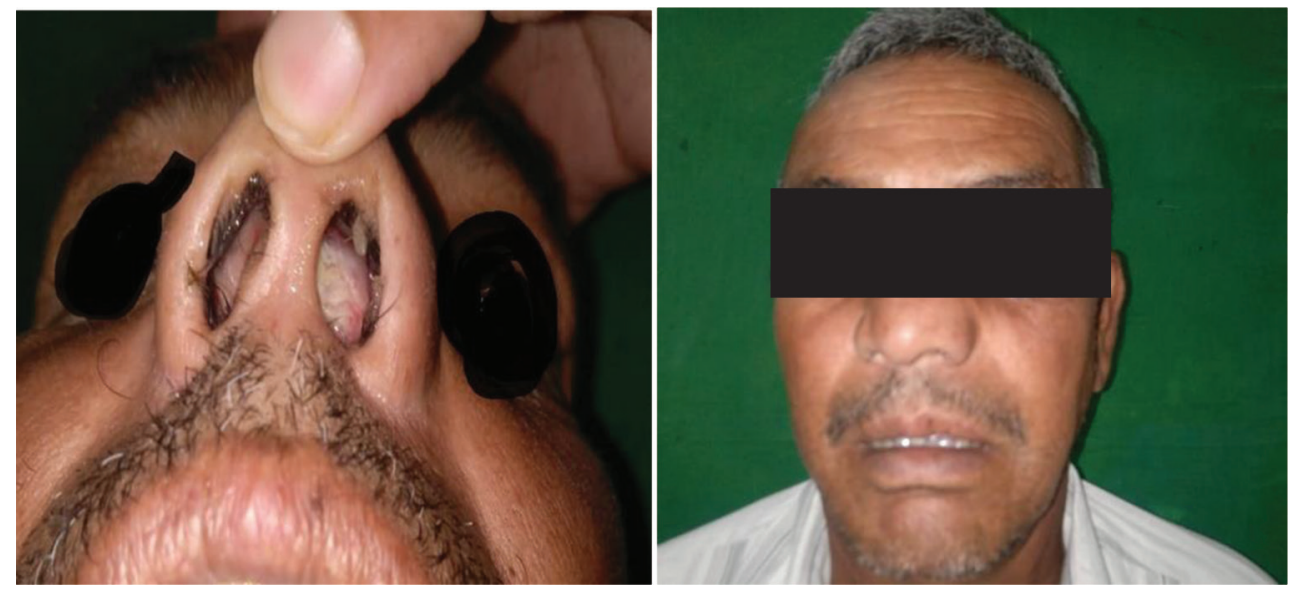

Fig. 1 Nasal mass and external deformity caused by the nasal mass.

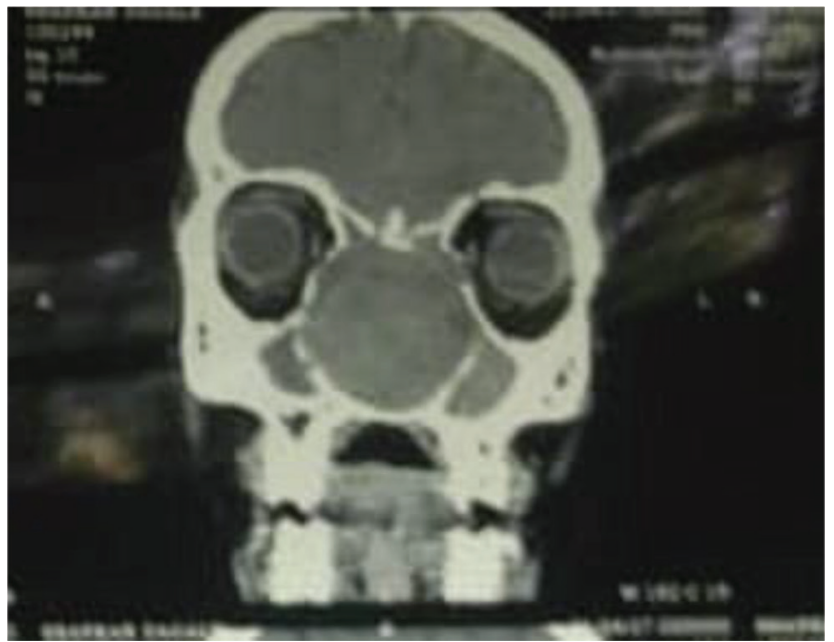

Fig. 2 Contrast-enhanced computed tomography image of the nose and paranasal sinus showing a mass in the left nasal cavity.

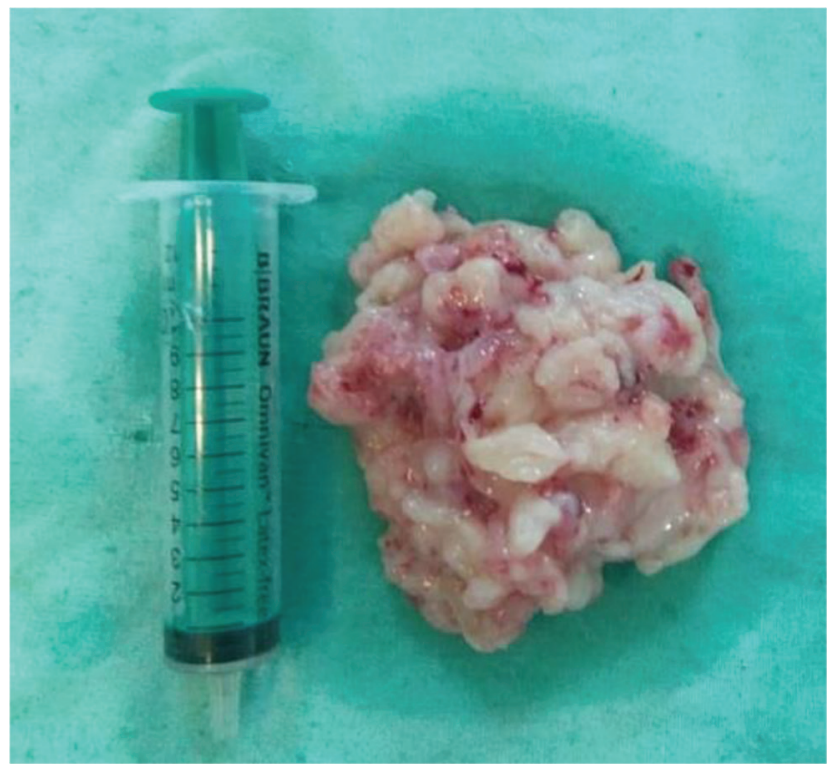

Fig. 3 Gross specimen of the nasal mass.
Since the tumor was benign in nature, decision of endoscopic surgical excision was made and the patient was planned for the surgery after due anesthesiologist and cardiac fitness. The tumor had a pedicle on the right nasal septum near the olfactory cleft, which we extirpated totally, leaving an adequate free margin. As the posterior part of the nasal septum was eroded, we performed posterior septectomy. The thick mucus in the ethmoidal sinus proved to be nonmalignant. Complete removal of the tumor in toto was possible with endoscopic surgery.

Intraoperative hemostasis was achieved, and the excised specimen was sent for histopathological examination. The report came to be confirmatory as of biopsy. The patient was followed up for 3 months. Diagnostic nasal endoscopy was performed every month, and there was no evidence of recurrence.

Pathological examination showed that the proliferation of spindle-shaped pericytes had surrounded the ecstatic blood vessels to create a so-called staghorn structure. The vimentin and actin studies were positive, whereas negative results were obtained from studies on CD-34, S-100 protein, and cytokeratin. The originating cells are thought to be meningeal capillary pericytes, Zimmerman pericytes, or precursor cells with angioblastic tendencies showing benign nature (-Fig. 4 ).

\section{Discussion}

In this report, we demonstrated that endoscopic surgery may be one of the useful treatments able to improve the prognosis of sinonasal HPC. Usually, HPC exhibits biologically malignant behavior, against which no effective combination of chemotherapy and radiotherapy has been established. Recently, the safety and efficacy of rIL-2 have been reported for angiosarcoma ${ }^{56}$ of the skin and renal cell carcinoma. ${ }^{7}$ rIL-2 is thought to attack the tumor cells directly as well as to activate the NK cells, triggering the anti-tumor effects. ${ }^{5,7}$ 


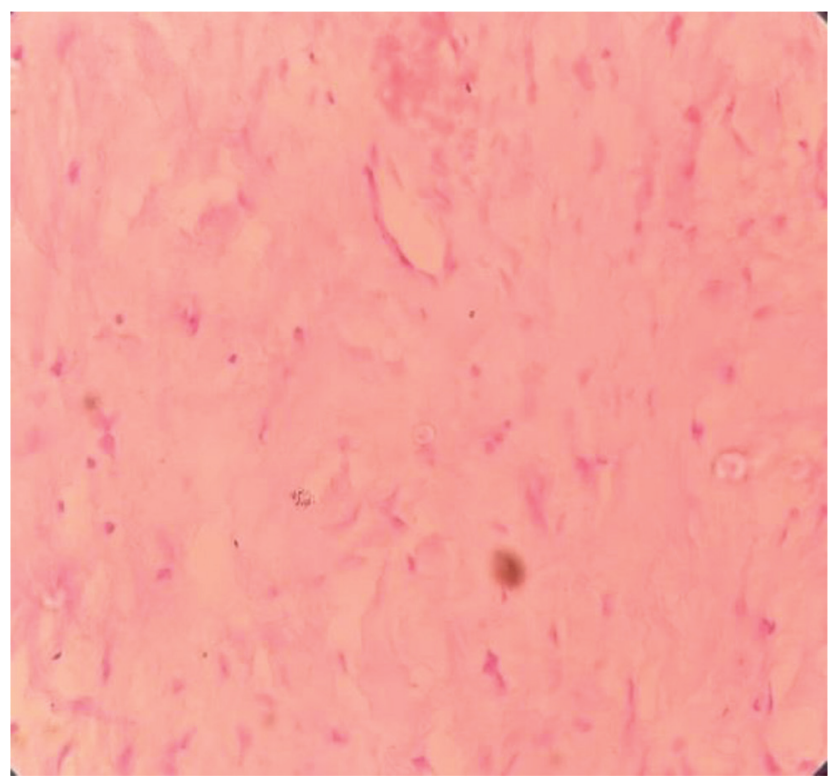

Fig. 4 Hematoxylin and eosin staining showing spindle cells and pericytes.

Patients receiving rIL-2 therapy may require management of severe side effects, especially cardiopulmonary and renal toxicities. ${ }^{8}$ Other minor side effects include fever elevation, flulike symptoms, fatigue, anorexia, and skin eruption. ${ }^{8,9}$ In our case, since the HPC was benign, we were able to avoid the dreaded complication of rIL-2, and complete excision of the tumor was possible with endoscopic surgery.

Sinonasal HPCs are rare, with less than 200 cases reported in the literature. ${ }^{1,10}$ HPCs are clinically classified into two types depending on the prognosis: malignant and benign. In the malignant type, the tumor tends to recur even after a wide surgical excision. On the other hand, in the benign type, the tumor does not recur. Although these categories exist, the natural history of the disease is not correlated with its histological grading but remains rather unpredictable. ${ }^{1}$

\section{Conclusion}

Since HPCs are uncommon vascular tumors and rarely occur in the nose or paranasal sinuses, further case studies are needed. Though treatment strategy has not been established, we can prevent patients from the facial scars and deformity by operating the benign conditions with endoscopic sinus surgery and keeping the external approach along the rIL-2 management for the malignant conditions.

\section{Conflict of Interest}

None declared.

\section{References}

1 Marianowski R, Wassef M, Herman P, Huy PT. Nasal haemangiopericytoma: report of two cases with literature review. J Laryngol Otol 1999;113(3):199-206

2 Hervé S, Abd Alsamad I, Beautru R, et al. Management of sinonasal hemangiopericytomas. Rhinology 1999;37(4):153-158

3 Pross HF, Lotzová E. Role of natural killer cells in cancer. Nat Immun 1993;12(4-5):279-292

4 Yang Q Goding SR, Hokland ME, Basse PH. Antitumor activity of NK cells. Immunol Res 2006;36(1-3):13-25

5 Fukushima K, Dejima K, Koike S, et al. A case of angiosarcoma of the nasal cavity successfully treated with recombinant interleukin-2. Otolaryngol Head Neck Surg 2006;134(5):886-887

6 Tanigawa T, Yajin K. A case of hemangiopericytoma from maxillary sinus. Otolaryngol Head Neck Surg (Tokyo) 1996;68:184-185

7 Atzpodien J, Hoffmann R, Franzke M, Stief C, Wandert T, Reitz M. Thirteen-year, long-term efficacy of interferon 2alpha and interleukin 2-based home therapy in patients with advanced renal cell carcinoma. Cancer 2002;95(5):1045-1050

8 Sharp E. Case management of the hospitalized patient receiving interleukin-2. Semin Oncol Nurs 1993;9(3, Suppl 1):14-19

9 Yang JC, Sherry RM, Steinberg SM, et al. Randomized study of high-dose and low-dose interleukin-2 in patients with metastatic renal cancer. J Clin Oncol 2003;21(16):3127-3132

10 Simmonds JC, Rebeiz EE. Surgical resection of sinonasal hemangiopericytoma involving anterior skull base: case reports and literature review. Am J Otolaryngol 2017;38(1):87-91 